\title{
Variant discovery in the sheep milk transcriptome using RNA sequencing
}

\author{
Aroa Suárez-Vega', Beatriz Gutiérrez-Gil', Christophe Klopp², Gwenola Tosser-Klopp ${ }^{3}$ and Juan José Arranz ${ }^{*}$
}

\begin{abstract}
Background: The identification of genetic variation underlying desired phenotypes is one of the main challenges of current livestock genetic research. High-throughput transcriptome sequencing (RNA-Seq) offers new opportunities for the detection of transcriptome variants (SNPs and short indels) in different tissues and species. In this study, we used RNA-Seq on Milk Sheep Somatic Cells (MSCs) with the goal of characterizing the genetic variation within the coding regions of the milk transcriptome in Churra and Assaf sheep, two common dairy sheep breeds farmed in Spain.

Results: A total of 216,637 variants were detected in the MSCs transcriptome of the eight ewes analyzed. Among them, a total of 57,795 variants were detected in the regions harboring Quantitative Trait Loci (QTL) for milk yield, protein percentage and fat percentage, of which $21.44 \%$ were novel variants. Among the total variants detected, 561 (2.52\%) and 1,649 (7.42\%) were predicted to produce high or moderate impact changes in the corresponding transcriptional unit, respectively. In the functional enrichment analysis of the genes positioned within selected QTL regions harboring novel relevant functional variants (high and moderate impact), the KEGG pathway with the highest enrichment was "protein processing in endoplasmic reticulum". Additionally, a total of 504 and 1,063 variants were identified in the genes encoding principal milk proteins and molecules involved in the lipid metabolism, respectively. Of these variants, 20 mutations were found to have putative relevant effects on the encoded proteins.

Conclusions: We present herein the first transcriptomic approach aimed at identifying genetic variants of the genes expressed in the lactating mammary gland of sheep. Through the transcriptome analysis of variability within regions harboring QTL for milk yield, protein percentage and fat percentage, we have found several pathways and genes that harbor mutations that could affect dairy production traits. Moreover, remarkable variants were also found in candidate genes coding for major milk proteins and proteins related to milk fat metabolism. Several of the SNPs found in this study could be included as suitable markers in genotyping platforms or custom SNP arrays to perform association analyses in commercial populations and apply genomic selection protocols in the dairy production industry.
\end{abstract}

Keywords: Dairy Sheep, Milk Somatic Cells, RNA-Seq, Transcriptome Variants

\section{Background}

The identification of genetic variation underlying desired phenotypes is one of the main challenges in current dairy genetic research. The higher content of sheep milk in total solids when compared to cow and goat milk favors its greater aptitude for cheese production [1]. Therefore, genetic variation within genes that influence

\footnotetext{
*Correspondence: jjarrs@unileon.es

${ }^{1}$ Departamento de Producción Animal, Facultad de Veterinaria, Universidad

de León, Campus de Vegazana s/n, León 24071, Spain

Full list of author information is available at the end of the article
}

the total solid content of milk is of crucial interest in dairy sheep breeding because this variability could be linked to milk composition, milk quality and cheese production.

Over the years, several studies on polymorphisms in ovine major milk proteins (caseins and whey proteins) have appeared due to the potential association of these polymorphisms with milk yield, milk composition and milk technological aspects [1-4]. Additionally, as the majority of dairy sheep traits are complex, research on dairy Quantitative Trait Loci (QTL) mapping has also 
been widely performed. To date, 1,336 sheep QTL influencing 212 different traits have been reported in a total of 119 publications (http://www.animalgenome.org/cgibin/QTLdb/index; accessed at 24 November 2016) [5]. In relation to milk traits, 242 QTL have been reported [5]. However, the traditional methodology used for QTL mapping with genome-wide sparse microsatellite markers or with low/middle density Single Nucleotide Polymorphism (SNP) genotyping platforms makes it difficult to identify the true causal mutations underlying these complex traits.

Over the last few years, the constant improvement of high-throughput sequencing platforms and the availability of genome sequencing data have facilitated the detection of a substantial number of genetic variants in livestock [6, 7]. The identification of this genomic variation is crucial to the rapid identification of mutations that compromise animal health and productivity but also to build a database of polymorphisms that could be used as molecular markers for more accurate genomic predictions and genome-wide association studies [6].

High-throughput transcriptome sequencing technology (RNA-Seq) has been developed to identify and quantify gene expression in different tissues $[8,9]$. Moreover, RNA-Seq also offers new opportunities for the efficient detection of transcriptome variants (SNPs and short indels) in different tissues and species $[10,11]$. In this way, when compared to whole genome sequencing, RNA-Seq offers a cheaper alternative to identifying variation and, possibly, discovering the causal mutations underlying the analyzed phenotypes $[12,13]$.

In this study, we used RNA-Seq on Milk Sheep Somatic Cells (MSCs) with the goal of characterizing the genetic variation in the coding regions of the milk transcriptome in two dairy sheep breeds, Churra and Assaf, that are commonly farmed in Spain. In addition to the general characterization of variations in the sheep milk transcriptome, we focused our analysis on the detection of variability within the coding regions harboring QTL for milk yield, fat percentage and protein percentage and in the genes codifying for major milk proteins and enzymes related to milk fat metabolism. Thus, this analysis has allowed for the discovery of functionally relevant variants within genes related to dairy production traits that could be exploited by dairy sheep breeding programs after further research confirms the possible associations with phenotypes of interest.

\section{Results and discussion}

\section{Sequencing and mapping}

Milk samples from eight ewes (four Churra and four Assaf) were collected at different lactation time points (days 10, 50, 120 and 150 after lambing). Based on the quality score of the RNA (RIN $>7$ ), we sequenced the
MSCs transcriptome from eight animals on days 10, 50 and 150 of lactation and from six animals on day 120 of lactation. A total of 1,116 million paired-end reads was obtained from the transcriptome sequencing of the 30 milk samples analyzed. An alignment of the reads to the Ovis aries Oar_v3.1 genome yielded a mean of $88.10 \%$ of the reads per RNA-Seq sample that aligned to unique locations in the ovine genome. After merging the replicates from the same animal at the different sampling time-points and marking the duplicates on the resulting merged bam files, we found that an average of 119.33 million non-duplicated paired-end reads per animal mapped to the Oarv3.1 genome assembly. General RNA-Seq metrics obtained with the RSeQC software [14] that consider the annotation bed file of the reference sheep genome are summarized in Table 1 . In our dataset of the sheep MSCs transcriptome, an average of 120.47 million tags per animal were defined. The term "tag" accounted for the number of times one read is spliced. The RSeQC program assigned an average of 110.08 million tags per merged sample to the annotated sheep genome regions. Therefore, approximately 10.39 million tags were not assigned to annotated regions, suggesting that approximately 10 million tags per sample mapped to intergenic regions. The comparative analysis performed in a previous study of the assembled transcripts of this RNA-Seq dataset with the ovine genome assembly Oar_v3.1 revealed that up to the $62 \%$ of the transcripts detected in the MSCs genome were intergenic [15]. These results reflect the incompleteness of the current annotation of the sheep transcriptome and presume the presence of non-annotated transcripts that

Table 1 Summary of sequencing results according to the annotation performed in this study of the MSC transcriptome based on the sheep genome reference Oar_v3.1

\begin{tabular}{|c|c|c|c|}
\hline \multicolumn{3}{|c|}{ Total Reads (paired end) } & \multirow{2}{*}{$\begin{array}{l}119325116 \\
120473958\end{array}$} \\
\hline Total Tags & & & \\
\hline \multicolumn{3}{|l|}{ Total Assigned } & 110083502 \\
\hline Group & Total_bases & Tag_count & Tags/kb \\
\hline $\mathrm{CDS}_{\text {_a }}^{\mathrm{a}}$ Exons & 32776750 & 65846229.88 & 2008.93 \\
\hline $5^{\prime} U_{T R}^{\mathrm{b}}$ _Exons & 3479917 & 960588.13 & 276.04 \\
\hline $3^{\prime} U_{T R}^{c}$ Exons & 8651433 & 4457991.13 & 515.29 \\
\hline Introns & 803999021 & 13554137.88 & 16.86 \\
\hline TSS__up_1 kb & 21995006 & 933617.50 & 42.45 \\
\hline TSS_up_5kb & 101300701 & 2521024.00 & 24.89 \\
\hline TSS_up_10kb & 187280303 & 3117103.63 & 16.64 \\
\hline TES $^{\mathrm{e}}$ _down_1 kb & 21770670 & 10545653.88 & 484.40 \\
\hline TES_down_5kb & 96011366 & 21156069.50 & 220.35 \\
\hline TES_down_10kb & 173072739 & 22147451.25 & 127.97 \\
\hline
\end{tabular}

${ }^{a}$ CDS Coding DNA sequence; ${ }^{\mathrm{b}} 5^{\prime} U T R$ leader untranslated sequence; ${ }^{\mathrm{c}} 3^{\prime} U T R$ trailer untranslated sequence; ${ }^{\mathrm{d}}$ TSS Transcription Start Site; ${ }^{\mathrm{e}}$ TES Transcription End Site 
could codify for novel proteins or constitute functional noncoding RNAs, like long noncoding RNAs (lncRNAs), microRNAs (miRNAs), short interfering RNAs (siRNAs), Piwi-interacting RNAs (piRNAs) or small nucleolar RNAs (snoRNAs). In the human genome the transcriptome functional non-coding elements have been estimated to constitute up to $98 \%$ of transcripts [16]. The identification of these functional elements in animals is one of the goals of the Functional Annotation of Animal Genomes (FAANG) project [17].

By focusing on assigned tags, as could be expected, the vast majority of tags mapped to coding genome regions. Specifically, we found an average of 65.85 million tags per animal, or $2008.93 \mathrm{tags} / \mathrm{kb}$ that mapped to CDSs (Table 1).

\section{Variant detection and functional annotation}

A total of 216,637 variants were detected in the MSCs transcriptome of the eight ewes analyzed after the variants were filtered (Table 2; Additional file 1). Of these variants, approximately the $78 \%$ were previously annotated in dbSNP (version 143). Among the total variants identified, 197,948 were SNPs and 18,689 were indels. The transition to transversion $(\mathrm{Ts} / \mathrm{Tv})$ ratio was 2.4 , which was slightly higher than the 2.0-2.2 genome-wide $\mathrm{Ts} / \mathrm{Tv}$ ratio reported in relation to human wholegenome sequence data [18]. However, this ratio is generally higher in exomes due to the increased presence of methylated cytosine in $\mathrm{CpG}$ dinucleotides in exonic regions [19].

Considering SNPs and Indels, the variant density across the genome (Fig. 1) showed a more or less uniform distribution, with three regions showing a high density of variants that should be noted (more than 800 variants $/ \mathrm{Mb}$ ). Two of these regions with high densities of variants were located on chromosome 20 (OAR20) at OAR20:26-27 Mb and OAR20:27-28 Mb, with 858 and 1321 variants/Mb, respectively. The Major Histocompatibility Complex (MHC) of sheep is located in a region of chromosome 20 [20] that corresponds to the $2 \mathrm{Mb}$ region with high variability detected in this study. This region on OAR20 was also identified to harbor a putative QTL for milk yield-related traits [21]. The other region with a high number of variants $(972$ variants/Mb) is located on OAR6 (OAR6:85-86 Mb) and is related to the genomic location of ovine genes coding for the milk caseins (OAR6: 85,087,000-85,318,000). The large number of variants positioned in this region could be due to the high transcription levels of caseins in the lactating mammary gland. The high transcription rate of the casein cluster region, with an average of 3.48 million of tags per kb of exon, refers to the transcription of both exons and the surrounding intronic regions. Hence, it is remarkable that a very high number of tags per kb of
Table 2 Summary statistics of the identified variants

\begin{tabular}{|c|c|c|}
\hline Fields & Counts SnpEff & Counts VEP \\
\hline Variants processed & 216637 & 212742 \\
\hline SNPS & 197948 & 195503 \\
\hline Insertions & 8603 & 7233 \\
\hline Deletions & 10086 & 9032 \\
\hline \multicolumn{3}{|l|}{ Effects by impact } \\
\hline $\mathrm{HIGH}$ & 2128 & 1891 \\
\hline MODERATE & 22440 & 22385 \\
\hline LOW & 43986 & 43667 \\
\hline MODIFIER & 312170 & 232768 \\
\hline \multicolumn{3}{|l|}{ Effects by type } \\
\hline 3_prime_UTR & 12940 & 12950 \\
\hline 5_prime_UTR & 1819 & 1824 \\
\hline downstream_gene & 113225 & 113207 \\
\hline frameshift & 1162 & 1096 \\
\hline inframe_deletion & 168 & 314 \\
\hline inframe_insertion & 127 & 229 \\
\hline intergenic_region & 96639 & 16991 \\
\hline intron & 59198 & 58408 \\
\hline missense & 21841 & 21824 \\
\hline non_coding_exon & 2002 & 1993 \\
\hline non_coding_transcript & 10 & 9492 \\
\hline splice_acceptor & 525 & 332 \\
\hline splice_donor & 594 & 371 \\
\hline splice_region & 2353 & 2187 \\
\hline start_lost & 16 & 28 \\
\hline stop_gained & 119 & 112 \\
\hline stop_lost & 28 & 30 \\
\hline stop_retained & 26 & 31 \\
\hline synonymous & 43003 & 43004 \\
\hline upstream_gene & 27952 & 27948 \\
\hline
\end{tabular}

intron was found in the casein cluster region (7011.22 tags per $\mathrm{kb}$ of intron) when compared with the average across the whole sheep genome (16.86 tags per kb of intron). Previous RNA-Seq analysis suggest that the pattern of the intronic sequence read coverage in RNA-Seq could be explained by an inefficient poly $(\mathrm{A})^{+}$purification [22], the presence of intronic reads flanked by poly $(\mathrm{A})^{+}$ stretches [23] or by transcripts undertaking splicing after polyadenylation [23].

The annotation analyses performed with SnpEff [24] and Variant Effect Predictor (VEP) [25] are summarized in Table 2. The number of variants processed with SnpEff was higher $(216,637)$ when compared to the variants processed with the VEP software $(212,742)$ because SnpEff performs the annotation of the variants present 


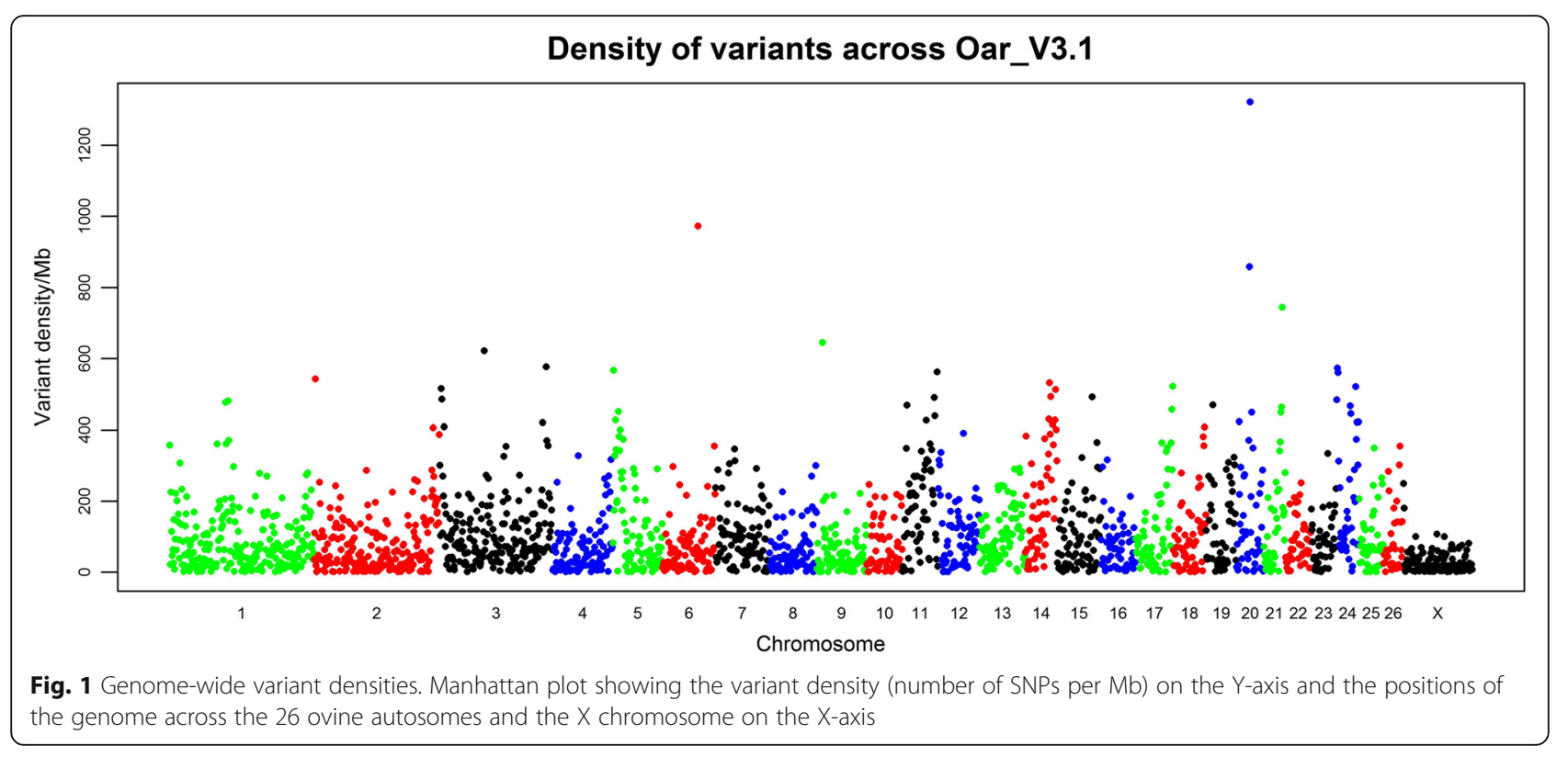

in the whole domestic sheep genome (Oar_v3.1), chromosomes and scaffolds, whereas VEP only annotates variants within ovine chromosomes. Variants were assigned to four types of biological impact based on the significance of the effect of the variant: high (e.g., frame shift, stop gain/loss, start loss, etc.); moderate (e.g., nonsynonymous coding changes, codon insertion/deletion, etc.); low (e.g., synonymous changes etc.); or modifier (used for terms with hard-to-predict effects and markers) (Table 2). The number of functional effects assigned was larger than the number of loci because the categories were not mutually exclusive. Among the total number of effects detected, the vast majority of the variants were predicted to have modifier impacts by both software programs (312,170 with SnpEff and 232,768 with VEP) (Table 2). This is because most of the variants detected were located in downstream gene regions (Table 2). Among the distribution of the variants by type of effect, the results of the two annotation tools were generally consistent (Table 2). Only two non-coding categories show marked discrepancies as follows: the variants annotated as intergenic regions and the variants annotated as non-coding transcript variants (Table 2). A higher number of variants were found by SnpEff than by VEP in intergenic regions (96,639 and 16,991, respectively), which could be due to the different performances of the annotation algorithms. The VEP software found a greater number of non-coding transcript variants than SnpEff (9,492 and 10 variants, respectively) because VEP annotates regulatory region variants without providing additional datasets to the software [25].

Among the results described in Table 2, it is remarkable the large proportion of variants identified within non-coding regions (e.g. downstream, intergenic, intronic variants) which could indicate the presence of variants in unannotated exons and/or noncoding but functionally transcribed genomic regions. As we have pointed above, the $62 \%$ of the transcripts detected within the ovine MSCs transcriptome were intergenic and moreover, the $11 \%$ were classified as potentially novel isoforms [15]. Therefore, the detection of variants out of known protein coding regions can be expected. Furthermore, these results agree with the results found in previous studies in cattle and human [26, 27]. However, further research needs to be done in the identification of transcriptome functional elements in livestock genomes to elucidate the potential role of the variants detected within no-coding regions.

\section{Variants in QTL regions}

A total of 57,795 variants were detected within the selected regions harboring QTL for milk yield, protein percentage and fat percentage. Among them, 78.56\% were mutations already described in SNPdb (version 143). Most QTL in dairy sheep have been mapped with low-density maps, resulting in the detection of the significant effect within large confidence intervals. Hence, the high amount of variants detected in this work within ovine QTL for dairy traits could be related to the low mapping resolution of many of the previously identified QTL effects.

Due to the large number of total variants found, we focused our further exploratory study on the novel variants detected. Among the 12,389 novel variants identified within QTL regions, 9,118 were SNPs, 2,161 were insertions and 1,110 were deletions. Approximately 
$82.15 \%$ of the identified novel variants were considered sequence modifiers; the remaining $(\sim 17,85 \%)$ were inferred to produce high impact (2.52\%), moderate impact $(7.42 \%)$ or low impact $(7.91 \%)$ changes in the corresponding transcriptional unit (Fig. 2).

Considering that the variants found within QTL regions may have been a consequence of selective pressures related to dairy production traits, we performed a functional enrichment analysis of the genes containing the variants with high and moderate functional impacts. For this analysis, we considered the variants that were classified as high and moderate impact variants (Fig. 2) by the two annotation software programs used, SnpEff [24] and VEP [25]. However, based on the large number of moderate missense variants identified by both programs (Fig. 2), we performed additional filtering to consider only the missense mutations predicted to be deleterious by SIFT [28], an external tool implemented in the VEP software that predicts the effects of an amino acid substitution on protein function. Hence, after discarding those variants predicted to be tolerated, a final total of 371 unique genes containing relevant functional variants (Additional file 2) were used to perform a functional enrichment analysis using the WEB-based Gene SeT AnaLysis Toolkit (WebGestalt) [29]. These genes were categorized by 14 enriched KEGG (Kyoto Encyclopedia of Genes and Genomes) pathway terms $\left(p_{a d j}<0.05\right)$ (Additional file 3). The highest enriched KEGG pathway was "protein processing in endoplasmic reticulum" with a $p_{\text {adj }}$ of $2.60 \mathrm{e}-05$. Metabolic processes in endoplasmic reticulum (ER) are associated with the synthesis and folding of membrane and secretory proteins as well as lipid synthesis. Under certain stress conditions (such as high levels of carbon-based molecules, free fatty acids, cytokines, and hypoxia), the accumulation of unfolded/misfolded proteins activates the ER stress signaling response [30,31]. The mammary gland faces high metabolic stress during lactation due to the elevated rates of protein and fat synthesis. In our study, the majority of the genes with relevant functional

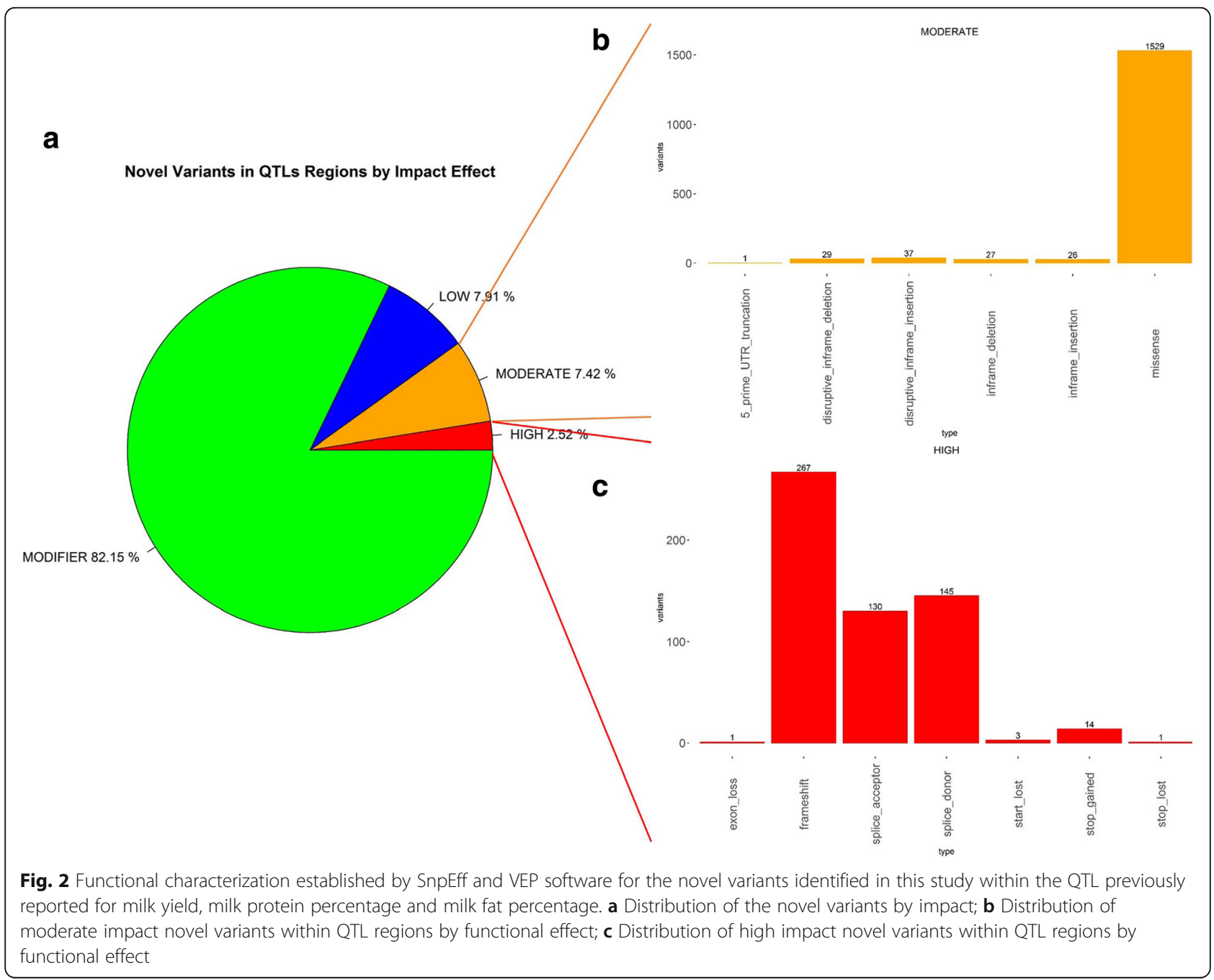


variants enriched in the KEGG pathway "protein processing in ER" were related to the ER stress response (CAPN2, HSP9OB1, PLAA, DERL2, DNAJB2, VCP, $U B Q L N 1$, SSR1). Mutations in these genes could be related to a different response of the overloaded ER in mutated animals during lactation, suggesting that these mutations could be a consequence of selective pressure for milk production traits. The high and moderate impact variants found in these genes and the animal genotypes for these variants are summarized in the additional information (Additional file 4).

Among the remaining enriched KEGG pathways $\left(p_{\text {adj }}<0.005\right)$ found in this analysis (Additional file 3), "Jak-STAT signaling pathway", "RNA transport" and "Fatty acid elongation" should be highlighted due to the putative influence of the genes within these pathways in milk yield or milk protein and fat content (see relevant variants and associated genes in Additional file 4). The Jak-STAT signaling pathway is directly implicated in milk protein expression by the mammary gland during lactation $[32,33]$. Among the variants found in the genes within this pathway, the variant found in the signal transducer and activator of transcription 4 (STAT4) gene is noteworthy because variants in the orthologous bovine gene have been significantly associated with milk yield and protein percentage $[34,35]$.

In the "RNA transport" pathway, it is worthwhile to highlight variants within the EIF4G3, EIF3I, and EIF3D genes. These three genes code for the eukaryotic translation initiation factors 4 Gamma 3, 3 Subunit I and 3 Subunit D, respectively. The binding of eIF4G to eIF3 is regulated by insulin via the association of mTOR with eIF3, which causes the initiation of translation in the mTOR signaling pathway [36, 37]. This pathway is implicated in the positive control of protein synthesis, and studies in ruminants have highlighted the crucial role of the mTOR signaling pathway in the regulation of milk protein synthesis [38].

The following two genes were enriched in the "Fatty acid elongation in mitochondria" KEGG pathway: $P P T 2$ and ACAA2. PPT2 is located within the ovine MHC region and encodes a member of the palmitoyl-protein thioesterase family, which has significant thioesterase activity against lipids with chain lengths of 10 or fewer carbons and 18 or more carbons [39]. The ACAA2 gene codes for the acetyl-CoA acyltransferase 2, a protein involved in lipid metabolism that catabolizes the last step in fatty acid $\beta$-oxidation. In Chios sheep, a single nucleotide polymorphism in $A C A A 2$ was identified and associated with the milk yield phenotype [40].

\section{Variants in sheep-cheese candidate genes Variants in genes related to milk protein content}

Variability related to milk protein content was evaluated in the genes codifying for major milk proteins, i.e., within the genes encoding caseins (casein $\alpha$-S1 (CSN1S1), casein $\alpha-S 2$ (CSN1S2), casein $\beta$ (CSN2), and casein $\kappa(C S N 3))$ and whey proteins ( $\alpha$-lactalbumin $(L A L B A)$ and $\beta$-lactoglobulin $(P A E P))$. After variant filtration a total of 504 variants were identified within these genes. Among these variants, 80 (15.9\%) variants were novel, and $424(84.1 \%)$ variants were previously annotated in SNPdb (version 143). Most of the detected variants in the major milk protein genes (452) were single nucleotide polymorphisms (SNPs). There were also 29 deletions and 23 insertions.

A high number of the variants found in the genes codifying for major milk proteins were positioned in introns (482). The large number of tags mapped to introns within the casein cluster, which was pointed above, together with the higher variability generally expected in non-coding regions may explain the high level of genetic variation identified in this region.

Among the variants detected in the coding regions by both software programs (SnpEff and VEP), we found one splice donor variant, which was classified as a high impact effect mutation, and ten missense variants. These mutations found within protein genes are summarized in Table 3. The splice donor variant found in the CSN1S2 gene is a novel variant that was detected in the two studied breeds (allele frequency of 0.625). This variant affects a putative splice donor site at the third intron of the CSN1S2 gene (GCA_000298735.1:6:85186875:G:A). Thus, this SNP could cause intron retention resulting in a novel isoform of CSN1S2, which should be confirmed by further research.

Missense variants in the ovine casein genes, which lead to amino acid changes in the protein products, comprise a group of SNPs that are of particular interest because some of these variants have been demonstrated to influence the composition and/or technological properties of milk (reviewed by Moioli et al. [41]). Among the missense variants detected in this study (Table 3), one was in CSN1S1, two were in CSN2 and three were in CSN1S2; no missense variants were found in CSN3. This result agrees with the fact that CSN3 is considered to be monomorphic in sheep [1]. Missense variants detected in the CSN1S2 gene are relevant due to their relationships with known protein alleles. The deleterious variant rs430397133 was detected in the CSN1S2 gene in one heterozygous Churra ewe (allele frequency of 0.125). The same animal was heterozygous for the other two missense variants found in CSN1S2, named rs424657035 and rs399378277, which were predicted to be tolerated. The mature protein of the known CSN1S2"B' variant harbors these three missense mutations [42]. The deleterious variant $r s 430397133$, which causes the Asp90Tyr substitution, is responsible for the higher isoelectric point of the $\mathrm{B}$ protein variant that allows for its 
Table 3 Functionally relevant variants in genes codifying for major milk proteins

\begin{tabular}{|c|c|c|c|c|c|}
\hline \multirow[t]{2}{*}{ Variant $^{a}$} & \multirow[t]{2}{*}{ Gene } & \multicolumn{2}{|c|}{ Allele Freq } & \multirow[t]{2}{*}{ Effect } & \multirow[t]{2}{*}{$\mathrm{AA}$} \\
\hline & & Assaf & Churra & & \\
\hline rs600923112 & PAEP & 0.25 & 0.5 & Missense-Deleterious & p.Gln167Leu \\
\hline rs600923112 & PAEP & 0.375 & 0 & Missense-Deleterious & p.GIn167Arg \\
\hline rs430610497 & PAEP & 0.375 & 0.5 & Missense-Tolerated & p.His36Tyr \\
\hline rs403176291 & $\angle A L B A$ & 0.125 & 0.5 & Missense-Deleterious & p.Val27Ala \\
\hline rs420959261 & CSNISI & 0.38 & 0.75 & Missense-Tolerated & p.Thr209lle \\
\hline rs416941267 & CSN2 & 0.625 & 0.25 & Missense-Tolerated & p.Leu212lle \\
\hline rs430298704 & CSN2 & 0 & 0.125 & Missense-Tolerated & p.Met199Va \\
\hline GCA_000298735.1:6:85186875:G:A & CSN1S2 & 0.625 & 0.625 & Splice donor & \\
\hline rs430397133 & CSN1S2 & 0 & 0.125 & Missense-Deleterious & p.Asp90Tyr \\
\hline rs424657035 & CSN1S2 & 0 & 0.25 & Missense-Tolerated & p.lle120Val \\
\hline rs399378277 & CSN1S2 & 0.125 & 0.75 & Missense-Tolerated & p.Arg176His \\
\hline
\end{tabular}

${ }^{a}$ For described variants $r$ identifier is indicated and novel variants are described with the unique ID "INSDC Genome accession:CHROM:POS:REF:ALT".

differentiation from CSN1S2*A [43]. An advantageous effect of $\mathrm{CSN} 1 \mathrm{~S} 2 * \mathrm{~B}$ in comparison to $\mathrm{CSN} 1 \mathrm{~S} 2 * \mathrm{~A}$ in terms of milk, fat and protein yield, and protein content has been reported [3]. In this study, we also found the variants responsible for $\alpha s 2-\mathrm{CN}$ protein alleles $\mathrm{G}$ (rs424657035) and G' (rs424657035 and rs399378277). However, at the protein level, the $G$ and $G$ ' alleles are hidden by the CSN1S2*A phenotype in isoelectric focusing [3].

In the CSN1S1 gene, we found a previously described missense variant ( $r$ 420959261). This SNP is responsible for the $p$.Thr209Ile substitution, which differentiates the protein variant $\mathrm{CSN} 1 \mathrm{~S} 1{ }^{*} \mathrm{C}$, the supposed ancestral variant, from CSN1S1*C" [44].

Two known SNPs, rs430298704 and rs416941267, were detected within the CSN2 gene. The rs430298704 SNP is a missense variant causing the substitution p.Met199Val which is classified as tolerated. This mutation causes the $A$ and $G$ protein alleles of $\beta$-casein. Corral et al. [45] found that in Merino sheep the GG genotype for this variant was associated with an increase in milk production, whereas the AA genotype was associated with an increase in protein and fat percentage. The rs416941267 is a missense variant causing the amino acid exchange p.Leu212Ile associated to the $\mathrm{CSN}^{*} \mathrm{X}$ protein allele described by Chessa et al. [46].

One already described missense SNP, rs403176291, was detected within the $L A L B A$ gene in both breeds. This mutation causes the amino acid change $p$.Val27Ala classified as deleterious by SIFT [28] and that has been suggested to be a Quantitative Trait Nucleotide (QTN) influencing milk protein percentage [47].

Regarding the PAEP ( $L G B)$ gene, which encodes the milk $\beta$-lactoglobulin protein, our analysis identified the missense variant (rs430610497) that differentiates protein alleles $\mathrm{A}$ and $\mathrm{B}$ of $\beta$-lactoglobulin [48, 49]. This mutation causes the substitution p.Tyr36His and was found in both breeds. A higher aptitude for cheese processing has been shown in AA ewes due to a shorter clotting time, better rate of curd firming and a higher cheese yield [2]. The $C$ allele of $\beta$-lactoglobulin [50] was not found in this study. This rare $C$ variant has been only found in few breeds, including Merinoland, Latxa, Carranzana, Spanish Merino, Serra da Estrela, White Merino, and Black Merino [2]. However, at position c.500 of the PAEP gene, we detected trialelic missense variants, rs600923112 and rs600923112, which cause two amino acid substitutions in the protein (p.Gln167Leu and p.Gln167Arg, respectively). The p.Gln167Leu amino acid change was found in the two studied breeds, whereas the p.Gln167Arg substitution was found only in Assaf sheep. These seem to be important mutations, as both amino acid changes are predicted to be deleterious by SIFT [28]. To our knowledge, these mutations are not related to described protein alleles in the $\beta$-lactoglobulin so further research should be conducted to elucidate their possible functional consequences.

\section{Variants in genes related to milk fat content}

To find variability in candidate genes related to milk fat content, we filtered the mutations positioned within a total of 17 genes (Table 4) that have been previously related to milk fat metabolism [51].

We detected a total of 1,063 variants in the transcriptomic regions containing the studied genes related to lipid metabolism. The majority of the variants within these genes $(953 ; 89.65 \%)$ were previously annotated in SNPdb (version 143). Among the variants detected, 990 were SNPs, 24 were insertions, and 49 were deletions. As these variants occurred in the genomic regions encoding caseins and whey proteins, the highest 
Table 4 Milk fat candidate genes considered in this study

\begin{tabular}{ll}
\hline Gene symbol & Description \\
\hline BTN1A1 & Butyrophilin Subfamily 1 Member A1 \\
ACACA & Acetyl-CoA Carboxylase Alpha \\
FABP3 & Fatty Acid Binding Protein 3 \\
CEL & Carboxyl Ester Lipase \\
ACSL1 & Acyl-CoA Synthetase Long-Chain Family Member 1 \\
LPL & Lipoprotein Lipase \\
ACSS2 & Acyl-CoA Synthetase Short-Chain Family Member 2 \\
XDH & Xanthine Dehydrogenase \\
GPAM & Glycerol-3-Phosphate Acyltransferase, Mitochondrial \\
DBI & Diazepam Binding Inhibitor, Acyl-CoA Binding Protein \\
VLDLR & Very Low Density Lipoprotein Receptor \\
DGAT1 & Diacylglycerol O-Acyltransferase 1 \\
PLIN2 & Perilipin 2 \\
SCD & Stearoyl-CoA Desaturase \\
LPIN1 & Lipin 1 \\
SLC27A6 & Solute Carrier Family 27 Member 6 \\
FASN & Fatty Acid Synthase \\
\hline
\end{tabular}

proportion of mutations were located within intronic regions (920; 86.39\%).

According to the functional effects by impact found in the fat-related genes, we identified four $(0.38 \%)$ variants with high impact, 27 (2.54\%) with moderate impact, 100 (9.39\%) with low impact and $934(87.7 \%)$ with a modifier impact. Among the moderate variants, we found a disruptive inframe deletion and 26 missense mutations, of which four were classified as deleterious by SIFT [28]. The functionally relevant variants within genes related to mammary gland fat metabolism are indicated in Table 5.

The highest number of functionally relevant variants were found in the $X D H$ gene. Two splice acceptor mutations and an inframe deletion were found in both breeds (Table 5 ). It should be noted that the inframe deletion (GCA_000298735.1:3:92239411:CCGCCCCTCTTCCC GGGCGCCCCCATCTTCTTTTCCA:C) was found in homozygosis in the eight ewes analyzed, which could mean that the $X D H$ sequence is not wellcharacterized at this genomic location. Moreover, two deleterious missense SNPs were found only in Assaf ewes (allele frequency of 0.125). $X D H$ encodes the xanthine dehydrogenase, a protein implicated in milk fat globule secretion [52]. Hence, mutations in this gene could alter the mechanisms underlying lipid droplet secretion.

PLIN2 encodes the perilipin 2/adipophilin protein. Adipophilin is reported to have a role in the packaging of triglycerides for secretion as milk lipids in the mammary gland [53]. Moreover, the absence of adipophilin has been associated with the formation of smaller intracellular fat globules [54]. The splice donor variant found within PLIN2 (GCA_000298735.1:2:87107748:C:A) gene is a novel variant that was detected in both breeds (allele frequency of 0.5 ). This variant affects a splice donor site at the first intron of the PLIN2 gene. Thus, this SNP could cause intron retention and a novel isoform.

A novel missense variant within the LPIN1 gene (GCA_000298735.1:3:20585665:C:T), causing the amino acid substitution p.Arg781Trp at the protein level, and classified as deleterious by SIFT [28], was found in heterozygosis in one Assaf sheep. LPIN1 encodes the lipin-1 protein, an enzyme implicated in triacylglycerol synthesis [32]. Additionally, a role for lipin-1 in the transcriptional regulation of other genes involved in milk lipid synthesis has been suggested in relation to the mTOR, PPAR $\alpha$ and PPAR $\gamma$ regulatory pathways [55-57].

In the FASN gene, we detected a known missense mutation ( $r$ 604791005) that causes the amino acid change p.Gly2312Ala. This polymorphism was found in

Table 5 Functionally relevant variants detected in the milk fat candidate genes considered in this study

\begin{tabular}{|c|c|c|c|c|c|}
\hline \multirow[t]{2}{*}{ Variant $^{\mathrm{a}}$} & \multirow[t]{2}{*}{ Gene } & \multicolumn{2}{|c|}{ Allele Freq } & \multirow[t]{2}{*}{ Effect } & \multirow[t]{2}{*}{$\mathrm{AA}$} \\
\hline & & Assaf & Churra & & \\
\hline GCA_000298735.1:2:87107748:C:A & PLIN2 & 0.5 & 0.5 & High-Splice donor & \\
\hline GCA_000298735.1:3:20585665:C:T & LPIN1 & 0.125 & 0 & Missense-Deleterious (0) & p.Arg781Trp \\
\hline GCA_000298735.1:3:92183603:G:T & $X D H$ & 0.5 & 0.5 & High-Splice aceptor & \\
\hline rs428221119 & $X D H$ & 0.25 & 0 & Missense-Deleterious (0.02) & p.Leu246Phe \\
\hline rs429850918 & $X D H$ & 0.25 & 0 & Missense-Deleterious (0) & p.Arg614Trp \\
\hline GCA_000298735.1:3:92217135:G:A & $X D H$ & 0.5 & 0.5 & High-Splice aceptor & \\
\hline $\begin{array}{l}\text { GCA_000298735.1:3:92239411: } \\
\text { CCGCCCCTCTTCCCGGGCGCCCCCATCTTCTTTTCCA:C }\end{array}$ & $X D H$ & 1 & 1 & Moderate-Inframe deletion & p.Pro1251_Phe1262del \\
\hline rs604791005 & FASN & 0 & 0.125 & $\begin{array}{l}\text { Missense-deleterious- } \\
\text { low_confidence }(0.04)\end{array}$ & p.Gly2312Ala \\
\hline GCA_000298735.1:26:13949071:C:T & ACSL1 & 0.5 & 0.5 & High-Splice donor & \\
\hline
\end{tabular}

${ }^{a}$ For described variants $r$ identifier is indicated and novel variants are described with the unique ID "INSDC Genome accession:CHROM:POS:REF:ALT" 
heterozygosis in one Churra ewe. FASN encodes a fatty acid synthase responsible for de novo fatty-acid biosynthesis in the mammary gland [58]. In cattle, several polymorphisms in this gene have been associated with milk fat content and fatty acid composition [59-64]. In Churra sheep, two QTL affecting capric acid and polyunsaturated fatty acid contents were mapped to the genomic region harboring the FASN gene [65], although the variability identified in this gene did not appear to be directly related to these QTL [65]. Therefore, the missense polymorphism described in this study should be further analyzed to assess its possible association with the QTL previously described in Churra sheep.

The splice donor variant found in the ACSL1 gene is a novel variant that was detected in both breeds (allele frequency of 0.5). This variant (GCA_000298735. 1:26:13949071:C:T) affects the first base of the 5 ' splice donor region of the second intron of ACSL1, which encodes an acyl-CoA synthetase long-chain family member 1. This protein is implicated in the activation of long chain fatty acids [32].

\section{Conclusions}

We present herein the first transcriptomic approach performed to identify the genetic variants of the lactating mammary gland in sheep. Through the transcriptome analysis of variability within regions harboring QTL for milk yield, protein percentage and fat percentage, we found several pathways and genes that could harbor mutations with relevant effects on dairy production traits. Moreover, remarkable variants were also found in candidate genes coding for major milk proteins and enzymes related to milk fat metabolism. Further research is required to estimate the allele frequencies and determine the phenotypic effects of the functionally relevant variants found through this RNA-Seq approach in commercial sheep populations. Additionally, several of the SNPs found in this study could be included as suitable markers in genotyping platforms or custom SNP-arrays to perform association analyses in commercial populations and apply genomic selection protocols in the dairy production industry.

\section{Methods}

\section{Animals and sampling}

For this study, a MSCs transcriptome dataset from Assaf and Spanish Churra dairy sheep breeds was used. The dataset is available in the Gene Expression Omnibus (GEO) database under the accession number GSE74825. The source of the animals and the sampling process protocol are described in detail in the related data descriptor manuscript [66]. The milk samples of eight healthy sheep (four Churra and four Assaf ewes) belonging to the commercial farm of the University of León were collected on days 10 (D10), 50 (D50), 120 (D120) and 150 (D150) after lambing. At each sampling timepoint, we collected $50 \mathrm{ml}$ of milk from each ewe one hour after the routine milking at 8 a.m. and ten minutes after the administration of five IUs of Oxytocin Facilpart (Syva, León, Spain). The time-point for milk collection was chosen to maximize the concentration of MSCs. Previous studies have indicated that the diurnal time point with the highest concentration of MSCs occurs one hour after milking [67]. Moreover, oxytocin was administered with the aim of stimulating its mechanical effect on myoepithelial contraction and thus the flattening of the alveolar lumen, which causes the release of residual post-milking milk containing a higher concentration of exfoliated MECs [68].

\section{Ethics statement}

All protocols involving animals were approved by the Animal Welfare Committee of the University of Leon, Spain, following the proceedings described in Spanish and EU legislations (Law 32/2007, R.D. 1201/2005, and Council Directive 2010/63/EU).

\section{Library preparation and sequencing}

Somatic cell separation and RNA extraction were performed as described by Suárez-Vega et al. (2016) [66]. The integrity of the RNA was assessed using an Agilent 2100 Bioanalyzer device (Agilent Technologies, Santa Clara, CA, USA). The RNA integrity value (RIN) of the samples ranged between 7.1 and 9. Paired-end libraries with fragments of $300 \mathrm{bp}$ were prepared using the TrueSeq RNA-Seq sample preparation Kit v2 (Illumina, San Diego, CA, USA). The fragments were sequenced on an Illumina Hi-Seq 2000 sequencer (Fasteris SA, Plan-lesOuates, Switzerland).

\section{Alignment, variant identification and annotation}

The read qualities of the RNA-Seq libraries were evaluated using FastQC [69]. Using the STAR aligner [70] the reads were mapped against the ovine genome assembly v.3.1. (Oar_v3.1 [71]). After the alignment, Samtools [72] was used to convert sam files to bam files and then to sort and merge the bam files from the same animal at different time-points. Metrics from the bam files were obtained with RSeQC software [14] based on the annotation bed file of the Oar_v3.1 sheep assembly obtained from the UCSC Genome Browser [73]. Then, Picard [74] was used to add read groups and mark duplicated reads on the merged bam files. SNP and Indel calling was performed using the Genome Analysis Toolkit (GATK, version 3.4.46) software package following GATK best practices [75]. To obtain high-quality variants, strict filter conditions were applied using vcffilter [76] and SnpSift [77] (Variation Quality (QUAL) >30, Mapping 
Quality (MQ) $>40$, Quality By Depth. (QD) $>5$, Fisher Strand (FS) $<60$ and a minimum Depth of coverage (DP) $>5$ in all the samples). The bcftools "annotate $-c$ ID" option [72] and the ovine reference vcf file downloaded from the Ensembl database (SNPdb-version 143) were used to annotate the known variants detected in our study.

Two software programs, SnpEff [24] and Variant Effect Predictor [25], were used to predict the functional consequences of the detected variants. SnpEff allows users to define specific intervals and customize the annotation of the variants. Considering that the final aim of this study is the characterization of the transcriptome variants that may be of special interest for the dairy industry, we used SnpEff to select (i) the variants included within previously reported sheep QTL studies for milk protein percentage, milk fat percentage and milk yield [5] and (ii) the variants included within candidate genes related to milk protein and fat content. The selection of the variants included in these two types of target regions (QTL and candidate genes) was performed according to the following criteria.

\section{Filtering variants in QTL regions affecting milk production traits}

The coordinates of the genomic regions containing the QTL related to milk protein percentage, milk fat percentage and milk yield, based on the annotation of the SheepQTLdb [5], were downloaded from the Ensembl database [71]. This information, provided as a bed file (Additional file 5), was used by the SnpEff software ( $-f i$ option) to retain only the variants matching the target QTL intervals from the total number of variants identified through the GATK protocol. Due to the high number of variants detected in the selected QTL regions $(57,795)$, those variants already described in the Ensembl database were filtered out using vcftools [78]. Among the novel variants, we selected those which were predicted by the two annotation analyses (SnpEff and VEP) to have relevant functional consequences. Thus, we retained those variants that were classified in terms of their functional consequences as "high" and "moderate" by the two different software programs. Due to the large number of variants classified as "moderate", within the moderate missense variants, we selected those predicted to be "deleterious" by the VEP option "-sift b" [25]. This option allows the use of the SIFT tool [28] for any of the variants annotated as missense. SIFT is an algorithm that predicts whether an amino acid substitution will have a deleterious effect on the protein function [28]. Finally, we extracted the names of the genes containing these functionally relevant mutations and used them to perform a functional enrichment analysis with the Webbased Gene Set Analysis Toolkit (WebGestalt) [29].

\section{Filtering variants on protein and fat candidate genes}

The candidate genes selected for a detailed analysis of their genetic variability in the studied dataset included those codifying for major milk constituent proteins (CSN1S1, CSN1S2, CSN2, CSN3, PAEP, LALBA) and 17 genes related to mammary gland lipid metabolism (Table 4). These genes were selected based on a previous study by our research group that evaluated the gene expression of candidate milk genes in the milk sheep transcriptome that affect cheese-related traits [51]. To obtain the variants within the target genes selected for the study, we used the $-f i$ option from SnpEff followed by a bed file with the coordinates of the selected genes (Additional files 6 and 7) and the -onlyTr option followed by a file with an ID list with the Ensembl transcripts name of the selected genes. From all the variants detected within the candidate cheese-yield genes, we focused further our analyses on those mutations that could have relevant consequences. Hence, the variants classified by the two software programs as having "high" and "moderate" functional impacts were selected.

\section{Additional files}

Additional file 1: Title of data: Variants detected within the sheep milk transcriptome. Description of data: Worksheet providing all the variants detected within the milk somatic cells transcriptome. (XLSX 60502 kb)

Additional file 2: Title of data: Genes in QTL regions containing relevant functional variants. Description of data: Worksheet providing the list of genes within QTL regions, which contain variants with functional interest. (XLSX $15 \mathrm{~kb}$ )

Additional file 3: Title of data: Results of the KEGG pathway enrichment analysis with the genes in QTL regions containing relevant functional variants. Description of data: Worksheet providing the results of the KEGG pathway enrichment analysis performed with the genes containing variants with functional interest. The file provides the enriched KEGG pathways, with the $p$-values and the genes grouped within each pathway. (XLSX $15 \mathrm{~kb}$ )

Additional file 4: Title of data: Functionally relevant variants found in the genes in "NOD-like receptor signaling pathway", "Protein processing in endoplasmic reticulum", "RNA tansport" and "Fatty acid elongation in mitochondria" KEGG pathways. Description of data: Worksheet providing the description and phenotypes of the functionally relevant variants found in the genes in "NOD-like receptor signaling pathway", "Protein processing in endoplasmic reticulum", "RNA tansport"and "Fatty acid elongation in mitochondria" KEGG pathways. (XLSX 15 kb)

Additional file 5: Title of data: Genomic regions containing the QTL related to milk protein percentage, milk fat percentage and milk yield. Description of data: Worksheet providing the coordinates of the genomic regions containing the QTL related to milk protein percentage, milk fat percentage and milk yield, based on the annotation of the SheepQTLdb. (XLSX $12 \mathrm{~kb}$ )

Additional file 6: Title of data: Coordinates of the milk protein genes genomic regions. Description of data: Worksheet providing the coordinates of the genomic regions containing the milk protein genes. (XLSX $9 \mathrm{~kb}$ )

Additional file 7: Title of data: Coordinates of the milk fat genes genomic regions. Description of data: Worksheet providing the coordinates of the genomic regions containing the genes related to milk fat metabolism. (XLSX $10 \mathrm{~kb}$ ) 


\section{Abbreviations}

ACAA2: Acetyl-CoA Acyltransferase 2; ACACA: Acetyl-CoA Carboxylase Alpha; ACSL1: Acyl-CoA Synthetase Long-Chain Family Member 1; ACSS2: Acyl-COA Synthetase Short-Chain Family Member 2; ALT: Alternative Allele; BTN1A1: Butyrophilin Subfamily 1 Member A1; CDS: Coding Sequence; CEL: Carboxyl Ester Lipase; CHROM: Chromosome; CSN1S1: Casein Alpha S1; CSN1S2: Casein Alpha S2; CSN2: Casein Beta; CSN3: Casein Kappa; D10: Day 10 after lambing; D120: Day 120 after lambing; D150: Day 150 after lambing; D50: Day 50 after lambing; DBI: Diazepam Binding Inhibitor, Acyl-CoA Binding Protein; dbSNP: NCBI database of genetic variation; DGAT1: Diacylglycerol OAcyltransferase 1; DP: Depth of Coverage; EIF3D: Eukaryotic Translation Initiation Factor 3 Subunit D; EIF31: Eukaryotic Translation Initiation Factor 3 Subunit I; EIF4G3: Eukaryotic Translation Initiation Factor 4 Gamma 3; ER: Endoplasmic Reticulum; EU: European Union; FAANG: Functional Annotation of Animal Genomes; FABP3: Fatty Acid Binding Protein 3; FASN: Fatty Acid Synthase; FS: Fisher Strand; GATK: Genome Analysis Toolkit; GEO: Gene Expression Omnibus; GPAM: Glycerol-3-Phosphate Acyltransferase, Mitochondrial; ID: Identifier; Indels: Insertions/Deletions; INSDC Genome accession: International Nucleotide Sequence Database Collaboration Genome accesion; IUs: International Units; kb: Kilobase; KEGG: Kyoto Encyclopedia of Genes and Genomes; LALBA: Lactalbumin Alpha; IncRNAs: long noncoding RNAs; LPIN1: Lipin 1; LPL: Lipoprotein Lipase; Mb: Megabase; MECs: Milk Epithelial Cells; MHC: Major Histocompatibility Complex; miRNAs: microRNAs; MQ: Mapping Quality; MSCs: Milk Somatic Cells; mTOR: Mechanistic Target Of Rapamycin; PAEP (LGB): Progestagen Associated Endometrial Protein (Beta-lactoglobulin); piRNAs: Piwi-interacting RNAs; PLIN2: Perilipin 2; POS: Position in the chromosome; PPARa: Peroxisome Proliferator Activated Receptor Alpha; PPARy: Peroxisome Proliferator Activated Receptor Gamma; PPT2: Palmitoyl-Protein Thioesterase 2; QD: Quality By Depth; QTL: Quantitative Trait Loci; QUAL: Variation Quality; REF: Reference Allele; RIN: RNA Integrity Number; RNA: Ribonucleic acid; RNA-Seq: RNA sequencing; SCD: Stearoyl-COA Desaturase; siRNAs: short interfering RNAs; SLC27A6: Solute Carrier Family 27 Member 6; snoRNAs: small nucleolar RNAs; SNP: Single Nucleotide Polymorphism; SnpEff: Genetic variant annotation and effect prediction toolbox; STAT4: Signal Transducer And Activator Of Transcription 4; Ts/TV: Transition to Transversion; VEP: Variant Effect Predictor; VLDLR: Very Low Density Lipoprotein Receptor; WebGestalt: WEB-based Gene SeT AnaLysis Toolkit; XDH: Xanthine Dehydrogenase

\section{Acknowledgements}

The support and availability to the computing facilities of the Foundation of Supercomputing Center of Castile and León (FCSCL) (http://www.fcsc.es) is greatly acknowledged.

\section{Funding}

This work is included in the framework of the project AGL2015-66035-R funded by the Spanish Ministry of Economy and Competitiveness (MINECO) and co-funded by European Regional Development Fund. B.G.G. is funded through the Spanish 'Ramón y Cajal' Program (RYC-2012-10230) from the MINECO.

\section{Availability of data and materials}

The datasets generated during and/or analyzed during the current study are available in the Gene Expression Omnibus (GEO) repository, under the accession number GSE74825. http://www.ncbi.nlm.nih.gov/geo/query/ acc.cgi?acc $=$ GSE74825

\section{Authors' contributions}

Conceived and designed the experiments: JJA. Performed the experiments: ASV, BGG and JJA. Analyzed the data: ASV, GTK and CK. Wrote the paper: ASV, BGG and JJA. All authors read and approved the final manuscript.

\section{Competing interests}

The authors declare that they have no competing interests.

\section{Consent for publication}

Not applicable.

\section{Ethics approval and consent to participate}

All protocols involving animals were approved by the Animal Welfare Committee of the University of Leon, Spain, following proceedings described in Spanish and EU legislations (Law 32/2007, R.D. 1201/2005, and Council Directive 2010/63/EU). All animals used in this study were handled in strict accordance with good clinical practices and all efforts were made to minimize suffering.

\section{Author details}

'Departamento de Producción Animal, Facultad de Veterinaria, Universidad de León, Campus de Vegazana s/n, León 24071, Spain. ${ }^{2}$ INRA, Plateforme bioinformatique Toulouse Midi-Pyrénées, UR875 Biométrie et Intelligence Artificielle, BP 5262731326 Castanet-Tolosan Cedex, France. ${ }^{3}$ GenPhySE, Université de Toulouse, INRA, INPT, ENVT, Castanet, Tolosan, France.

\section{Received: 25 August 2016 Accepted: 10 February 2017}

Published online: 15 February 2017

\section{References}

1. Selvaggi M, Laudadio V, Dario C, Tufarelli V. Investigating the genetic polymorphism of sheep milk proteins: a useful tool for dairy production. J Sci Food Agric. 2014;94:3090-9.

2. Selvaggi M. $\beta$-Lactoglobulin gene polymorphisms in sheep and effects on milk production traits: A Review. Adv Anim Vet Sci. 2015;3:478-84.

3. Giambra IJ, Brandt H, Erhardt G. Milk protein variants are highly associated with milk performance traits in East Friesian Dairy and Lacaune sheep. Small Rumin Res. 2014;121:382-94.

4. Amigo L, Recio I, Ramos M. Genetic polymorphism of ovine milk proteins: its influence on technological properties of milk- a review. Int Dairy J. 2000; 10:135-49.

5. Hu Z-L, Park CA, Reecy JM. Developmental progress and current status of the Animal QTLdb. Nucleic Acids Res Oxford University Press. 2016; 44:D827-33.

6. Daetwyler HD, Capitan A, Pausch H, Stothard P, van Binsbergen R, Brøndum $R F$, et al. Whole-genome sequencing of 234 bulls facilitates mapping of monogenic and complex traits in cattle. Nat Genet. 2014;46:858-65.

7. Georges M. Towards sequence-based genomic selection of cattle. Nat Genet. 2014:46:807-9.

8. Mortazavi A, Williams BA, McCue K, Schaeffer L, Wold B. Mapping and quantifying mammalian transcriptomes by RNA-Seq. Nat. Methods. 2008;5: 621-8.

9. Wang Z, Gerstein M, Snyder M. RNA-Seq: a revolutionary tool for transcriptomics. Nat Rev Genet. 2009;10:57-63.

10. Cánovas A, Rincon G, Islas-Trejo A, Wickramasinghe S, Medrano JF. SNP discovery in the bovine milk transcriptome using RNA-Seq technology. Mamm Genome. 2010;21:592-8.

11. Cox LA, Glenn JP, Spradling KD, Nijland MJ, Garcia R, Nathanielsz PW, et al. A genome resource to address mechanisms of developmental programming: determination of the fetal sheep heart transcriptome. J Physiol. 2012;590:2873-84.

12. Hudson NJ, Dalrymple BP, Reverter A, Hudson N, Reverter A, Dalrymple B, et al. Beyond differential expression: the quest for causal mutations and effector molecules. BMC Genomics. 2012;13:356.

13. Suárez-Vega A, Gutiérrez-Gil B, Benavides J, Perez V, Tosser-Klopp G, Klopp C et al. Combining GWAS and RNA-Seq approaches for detection of the causal mutation for hereditary junctional epidermolysis bullosa in sheep. PLoS One. 2015;10:e0126416.

14. Wang L, Wang S, Li W. RSeQC: quality control of RNA-seq experiments. Bioinformatics. 2012;28:2184-5.

15. Suárez-Vega A, Gutiérrez-Gil B, Klopp C, Robert-Granie C, Tosser-Klopp G, Arranz JJ. Characterization and comparative analysis of the milk transcriptome in two dairy sheep breeds using RNA sequencing. Sci Rep. 2015;5:18399.

16. Peschansky VJ, Wahlestedt C. Non-coding RNAs as direct and indirect modulators of epigenetic regulation. Epigenetics. 2014;9:3-12.

17. Andersson L, Archibald AL, Bottema CD, Brauning R, Burgess SC, Burt DW, et al. Coordinated international action to accelerate genome-to-phenome with FAANG, the Functional Annotation of Animal Genomes project. Genome Biol BioMed Central. 2015;16:57.

18. DePristo MA, Banks E, Poplin R, Garimella KV, Maguire JR, Hartl C, et al. A framework for variation discovery and genotyping using next-generation DNA sequencing data. Nat. Genet. 2011;43:491-8.

19. Hodges E, Smith AD, Kendall J, Xuan Z, Ravi K, Rooks M, et al. High definition profiling of mammalian DNA methylation by array capture and 
single molecule bisulfite sequencing. Genome Res Cold Spring Harbor Lab. 2009;19:1593-605.

20. Dukkipati VSR, Blair HT, Garrick DJ, Murray A. "Ovar-Mhc" - ovine major histocompatibility complex: structure and gene polymorphisms. Genet Mol Res. 2006;:581-608

21. Mateescu RG, Thonney ML. Genetic mapping of quantitative trait loci for milk production in sheep. Anim Genet. 2010;41:460-6.

22. Wetterbom A, Ameur A, Feuk L, Gyllensten U, Cavelier L, Chen F, et al. Identification of novel exons and transcribed regions by chimpanzee transcriptome sequencing. Genome Biol. 2010;11:R78.

23. Ameur A, Zaghlool A, Halvardson J, Wetterbom A, Gyllensten U, Cavelier L, et al. Total RNA sequencing reveals nascent transcription and widespread co-transcriptional splicing in the human brain. Nat Struct Mol Biol. 2011;18: $1435-40$.

24. Cingolani P, Platts A, Wang LL, Coon M, Nguyen T, Wang L, et al. A program for annotating and predicting the effects of single nucleotide polymorphisms, SnpEff: SNPs in the genome of Drosophila melanogaster strain w1118; iso-2; iso-3. Fly (Austin). 2012;6:80-92.

25. McLaren W, Gil L, Hunt SE, Riat HS, Ritchie GRS, Thormann A, et al. The Ensembl Variant Effect Predictor. Genome Biol. 2016;17:122.

26. Djari A, Esquerré D, Weiss B, Martins F, Meersseman C, Boussaha M, et al. Gene-based single nucleotide polymorphism discovery in bovine muscle using next-generation transcriptomic sequencing. BMC Genomics. 2013;14:307.

27. Piskol R, Ramaswami G, Li JB. Reliable identification of genomic variants from RNA-Seq data. Am J Hum Genet. 2013;93:641-51.

28. Kumar P, Henikoff S, Ng PC. Predicting the effects of coding nonsynonymous variants on protein function using the SIFT algorithm. Nat Protoc. 2009:4:1073-81.

29. Wang J, Duncan D, Shi Z, Zhang B. WEB-based GEne SeT AnaLysis Toolkit (WebGestalt): update 2013. Nucleic Acids Res. 2013;41:W77-83.

30. Fonseca SG, Gromada J, Urano F. Endoplasmic reticulum stress and pancreatic $\beta$-cell death. Trends Endocrinol Metab. 2011;22:266-74.

31. Gopinath RK, Leu J-Y. Hsp90 maintains proteostasis of the galactose utilization pathway to prevent cell lethality. Mol Cell Biol. 2016;36:1412-24.

32. Bionaz $\mathrm{M}$, Loor JJ. Gene networks driving bovine milk fat synthesis during the lactation cycle. BMC Genomics. 2008;9:366.

33. Rupp R, Senin P, Sarry J, Allain C, Tasca C, Ligat L, et al. A point mutation in Suppressor Of Cytokine Signalling 2 (Socs2) increases the susceptibility to inflammation of the mammary gland while associated with higher body weight and size and higher milk production in a sheep model. PLoS One. 2015. doi:10.1371/journal.pgen.1005629.

34. Zhang F, Huang J, Li Q, Ju Z, Li J, Shi F, et al. Novel single nucleotide polymorphisms (SNPs) of the bovine STAT4 gene and their associations with production traits in Chinese Holstein cattle. African J Biotechnol. 2010; 9:4003-8.

35. Song $X M$, Zhang $L$, Jiang JF, Shi $F X$, Jiang $Y Q$. An Sdul polymorphism at intron 20 of the Chinese Holstein cow STAT4 gene and its effect on milk performance traits. Genet Mol Res. 2013;12:1593-602.

36. LeFebvre AK, Korneeva NL, Trutschl M, Cvek U, Duzan RD, Bradley CA, et al. Translation initiation factor elF4G-1 binds to elF3 through the elF3e subunit. J Biol Chem. 2006;281:22917-32.

37. Laplante M, Sabatini DM. mTOR signaling at a glance. J Cell Sci. 2009;122: 3589-94.

38. Bionaz M, Loor JJ. Gene networks driving bovine mammary protein synthesis during the lactation cycle. Bioinforma Biol Insights. 2011;5:83-985.

39. Calero G, Gupta P, Nonato MC, Tandel S, Biehl ER, Hofmann SL, et al. The crystal ctructure of Palmitoyl Protein Thioesterase-2 (PPT2) reveals the basis for divergent substrate specificities of the two lysosomal thioesterases, PPT1 and PPT2. J Biol Chem. 2003;278:37957-64.

40. Orford M, Hadjipavlou G, Tzamaloukas O, Chatziplis D, Koumas A, Mavrogenis A, et al. A single nucleotide polymorphism in the acetylcoenzyme A acyltransferase 2 (ACAA2) gene is associated with milk yield in Chios sheep. J Dairy Sci. 2012;95:3419-27.

41. Moioli B, D'Andrea M, Pilla F. Candidate genes affecting sheep and goat milk quality. Small Rumin Res. 2007;68:179-92.

42. Tetens JL, Drögemüller C, Thaller G, Tetens J. DNA-based identification of novel ovine milk protein gene variants. Small Rumin Res. 2014;121:225-31.

43. Picariello G, Rignanese D, Chessa S, Ceriotti G, Trani A, Caroli A, et al. Characterization and genetic study of the ovine alphaS2-casein (CSN1S2) allele B. Protein J. 2009;28:333-40
44. Ceriotti G, Chessa S, Bolla P, Budelli E, Bianchi L, Duranti E, et al. Single Nucleotide polymorphisms in the ovine casein genes detected by polymerase chain reaction-single strand conformation polymorphism. J Dairy Sci. 2004;87:2606-13.

45. Corral JM, Padilla JA, Izquierdo M. Associations between milk protein genetic polymorphisms and milk production traits in Merino sheep breed. Livest Sci. 2010;129:73-9.

46. Chessa S, Rignanese D, Berbenni M, Ceriotti G, Martini M, Pagnacco G, et al. New genetic polymorphisms within ovine $\beta$ - and aS2-caseins. Small Rumin Res. 2010;88:84-8.

47. Garcia-Gamez E, Gutierrez-Gil B, Sahana G, Sanchez JP, Bayon Y, Arranz JJ. GWA analysis for milk production traits in dairy sheep and genetic support for a QTN influencing milk protein percentage in the LALBA gene. PLoS One. 2012;7:e47782.

48. Ali S, McClenaghan M, Simons JP, Clark AJ. Characterisation of the alleles encoding ovine B-lactoglobulins A and B. Gene. 1990;91:201-7.

49. Bell $K$, McKenzie HA. The whey proteins of ovine milk: $\beta$-lactoglobulins $A$ and B. Biochim Biophys Acta. 1967;147:123-34.

50. Erhardt G. Evidence for a third allele at the $\beta$-lactoglobulin ( $\beta$-Lg) locus of sheep milk and its occurrence in different breeds. Anim Genet. 2009;20:197-204.

51 Suárez-Vega A, Gutiérrez-Gil B, Arranz JJ. Transcriptome expression analysis of candidate milk genes affecting cheese-related traits in 2 sheep breeds. J Dairy Sci. 2016;99:6381-90.

52 McManaman JL, Russell TD, Schaack J, Orlicky DJ, Robenek H. Molecular determinants of milk lipid secretion. J Mammary Gland Biol Neoplasia. 2007; 12:259-68.

53 Russell TD, Palmer CA, Orlicky DJ, Bales ES, Chang BH-J, Chan L, et al. Mammary glands of adipophilin-null mice produce an amino-terminally truncated form of adipophilin that mediates milk lipid droplet formation and secretion. J Lipid Res. 2008;49:206-16.

54 Russell TD, Schaack J, Orlicky DJ, Palmer C, Chang BH-J, Chan L, et al. Adipophilin regulates maturation of cytoplasmic lipid droplets and alveolae in differentiating mammary glands. J Cell Sci. 2011;124:3247-53.

55 Huffman TA, Mothe-Satney I, Lawrence JC. Insulin-stimulated phosphorylation of lipin mediated by the mammalian target of rapamycin. Proc Natl Acad Sci. 2002;99:1047-52.

56 Finck BN, Gropler MC, Chen Z, Leone TC, Croce MA, Harris TE, et al. Lipin 1 is an inducible amplifier of the hepatic PGC-1a/PPARa regulatory pathway. Cell Metab. 2006:4:199-210.

57 Reue K, Zhang P. The lipin protein family: Dual roles in lipid biosynthesis and gene expression. FEBS Lett. 2008;582:90-6.

58 Smith S. The animal fatty acid synthase: one gene, one polypeptide, seven enzymes. FASEB J. 1994;8:1248-59.

59 Roy R, Ordovas L, Zaragoza P, Romero A, Moreno C, Altarriba J, et al. Association of polymorphisms in the bovine FASN gene with milk-fat content. Anim Genet. 2006;37:215-8.

60 Morris CA, Cullen NG, Glass BC, Hyndman DL, Manley TR, Hickey SM, et al. Fatty acid synthase effects on bovine adipose fat and milk fat. Mamm Genome. 2007;18:64-74.

61 Zhang S, Knight TJ, Reecy JM, Beitz DC. DNA polymorphisms in bovine fatty acid synthase are associated with beef fatty acid composition. Anim Genet. 2008;39:62-70.

62 Abe T, Saburi J, Hasebe H, Nakagawa T, Misumi S, Nade T, et al. Novel mutations of the FASN gene and their effect on fatty acid composition in Japanese Black Beef. Biochem Genet. 2009;47:397-411.

63 Schennink A, Bovenhuis H, Léon-Kloosterziel KM, Van Arendonk JAM, Visker MHPW. Effect of polymorphisms in the FASN, OLR1, PPARGC1A, PRL and STAT5A genes on bovine milk-fat composition. Anim Genet. 2009;40:909-16.

64 Matsumoto H, Inada S, Kobayashi E, Abe T, Hasebe H, Sasazaki S, et al. Identification of SNPs in the FASN gene and their effect on fatty acid milk composition in Holstein cattle. Livest Sci. 2012;144:281-4.

65 García-Fernández M, Gutiérrez-Gil B, García-Gámez E, Sánchez JP, Arranz JJ. The identification of QTL that affect the fatty acid composition of milk on sheep chromosome 11. Anim Genet. 2010;41:324-8.

66 Suárez-Vega A, Gutiérrez-Gil B, Klopp C, Tosser-Klopp G, Arranz J-J, Marioni JC, et al. Comprehensive RNA-Seq profiling to evaluate lactating sheep mammary gland transcriptome. Sci Data. 2016;3:160051.

67 Gonzalo C, Carriedo JA, Gomez JD, Gomez LD, San Primitivo F. Diurnal variation in the somatic cell count of ewe milk. J Dairy Sci. 1994;77:1856-9.

68 Peris C, Molina P, Fernandez N, Rodriguez M, Torres A. Variation in somatic cell count, California mastitis test, and electrical conductivity among various fractions of ewe's milk. J Dairy Sci. 1991;74:1553-60. 
69 Andrews S. FastQC A Quality Control tool for High Throughput Sequence Data. Babraham Bioinformatics. 2012. http://www.bioinformatics.babraham ac.uk/projects/fastqc/. Accessed 24 Aug 2016.

70 Dobin A, Davis CA, Schlesinger F, Drenkow J, Zaleski C, Jha S, et al. STAR: ultrafast universal RNA-seq aligner. Bioinformatics. 2013;29:15-21.

71 International Sheep Genome Consortium. Ovis aries Oar_v3.1, INSDC Assembly. Ensembl database. 2012. http://www.ensembl.org/Ovis_aries/lnfo/ Index. Accessed 24 Aug 2016

72 Li H, Handsaker B, Wysoker A, Fennell T, Ruan J, Homer N, et al. The Sequence Alignment/Map format and SAMtools. Bioinformatics. 2009;25: 2078-9.

73 Karolchik D, Hinrichs AS, Furey TS, Roskin KM, Sugnet CW, Haussler D, et al. The UCSC Table Browser data retrieval tool. Nucleic Acids Res. 2004;32: 493D-6D.

74 Wysoker A, Tibbetts K, McCowan M, Homer N, Fennell T. Picard Tools. http://broadinstitute.github.io/picard/ (2010). Accessed 24 Aug 2016.

75 McKenna A, Hanna M, Banks E, Sivachenko A, Cibulskis K, Kernytsky A, et al. The genome analysis toolkit: a MapReduce framework for analyzing nextgeneration DNA sequencing data. Genome Res. 2010;20:1297-303.

76 Garrison E. Vcflib: A C++ library for parsing and manipulating VCF files. Available from: https://github.com/vcflib/vcflib\#tvcflib (2012). Accessed 24 Aug 2016.

77 Cingolani P, Patel VM, Coon M, Nguyen T, Land SJ, Ruden DM, et al. Using Drosophila melanogaster as a model for genotoxic chemical mutational studies with a new program. SnpSift Front Genet. 2012;3:35.

78 Danecek P, Auton A, Abecasis G, Albers CA, Banks E, DePristo MA, et al. The variant call format and VCFtools. Bioinformatics. 2011;27:2156-8.

\section{Submit your next manuscript to BioMed Central and we will help you at every step:}

- We accept pre-submission inquiries

- Our selector tool helps you to find the most relevant journal

- We provide round the clock customer support

- Convenient online submission

- Thorough peer review

- Inclusion in PubMed and all major indexing services

- Maximum visibility for your research

Submit your manuscript at www.biomedcentral.com/submit
Biomed Central 